\title{
$33 \mathrm{~nm}$ wavelength tuning of a $1550 \mathrm{~nm}$ VCSEL in CW operation based on the liquid crystal micro-cells technology
}

\author{
$\underline{\text { C. Paranthoen }}{ }^{1,}$, C. Levallois ${ }^{1, *}$, B. Boisnard ${ }^{2}$, T. Camps ${ }^{2}$, J.-B. Doucet ${ }^{2}$, K. Tavernier ${ }^{1}$, N. Chevalier ${ }^{1}$, S. Bouchoule ${ }^{3}$, L. \\ Dupont $^{4}$, M. Alouini ${ }^{1}$, and V. Bardinal ${ }^{2}$ \\ ${ }^{11}$ Univ Rennes, INSA Rennes, CNRS, Institut FOTON - UMR 6082, F-35000 Rennes, France \\ ${ }^{2}$ Univ Toulouse, CNRS, LAAS, 7 Ave Colonel Roche, F-31400 Toulouse, France \\ ${ }^{3}$ C2N, CNRS, Université Paris-Sud, 91360 Marcoussis \\ ${ }^{4}$ IMT Atlantique, Optics Department, 655 Avenue du Technopôle, 29200 Plouzané, France \\ *Email: Cyril.paranthoen@insa-rennes.fr
}

\begin{abstract}
We present CW operation of a tunable InP based Vertical Cavity Surface Emitting Laser, integrating a liquid crystal (LC) micro-cell. In comparison with previous work, a larger $33 \mathrm{~nm}$ (vs $23 \mathrm{~nm}$ ) tuning and stable operation are obtained, according to major improvements presented in this paper.
\end{abstract}

\section{Introduction}

Miniaturized tunable photonic devices are of great interest for applications in wavelength division multiplexing systems, metrology, spectroscopy and sensing applications. For a widespread deployment, such systems have to be compact, dynamically reconfigurable in wavelength, and with a low power consumption. To achieve such devices, many studies have been conducted in the past using MEMS technologies [1]. However, MEMS solutions may have some drawbacks related to power consumption, high driving voltage or fragility of the MEMS structure. As an alternative to these MEMS approaches, the use of the well-known and mature liquid crystals (LC) material within a monolithic microcavity structure has been proposed [2]. In this case a large refractive index variation is obtained with a moderate driving voltage leading to a low power consumption since leakage currents through LC layers are extremely low. We have already developed this technology by fabricating LC micro-cells, and we have been able to realize and demonstrate passive optical filtering, and wavelength selective tunable integrated photodetector with large tuning range as high as 100-150 nm [3], [4]. More recently, we demonstrated for the first time CW operation of a tunable VCSEL integrating this LC micro-cell concept, presenting however a lower than expected $23.5 \mathrm{~nm}$ tuning range, and suffering from polarization instabilities [5].

\section{VCSEL fabrication}

The LC based tunable VCSEL we already proposed and demonstrated for optical pumping is presented on the insert of the Fig. 1. It mainly consists of two parts. The first one is the half-VCSEL processing. It consists in the growth of 9 strained InGaAsP quantum wells (SQW) on $\mathrm{InP}(100)$ substrate and distributed over three field anti-nodes. A SiNx/Si Bragg reflector (Bottom DBR) is then deposited, and this half VCSEL is then bonded on a silicon substrate. The second part concerns the fabrication of a LC micro-cell. It starts with the deposition of a $\mathrm{SiO}_{2} / \mathrm{TiO}_{2}$ Bragg reflector (top DBR) on a glass substrate initially covered by a conductive ITO layer. At this stage a nanograting is imprinted in SU8 photoresist for LC anchoring and alignment on the surface of this DBR. Both parts are then sealed together after the definition of micro-sized cell in $2.5 \mu \mathrm{m}$ thick of Perminex photoresist. The process ends up by the insertion of LC under vaccum. More details about this process can be found in reference [3]. Although this first realization enabled to demonstrate $\mathrm{CW}$ operation at $1550 \mathrm{~nm}$, a limited tuning range $(23.5 \mathrm{~nm})$ was obtained, and was mainly related to temperature effect inducing a collapse of the LC index variation and also because some instabilities in the output VCSEL polarization state [5]. To overcome this, we propose here two main modifications in the technology. The first one is to replace the SQW active layer by a hybrid active layer, which is characterized by 6 SQW distributed over two anti-nodes, and 6 quantum dashes (QDHs) layers on the third anti-node. These QDHs are expected to bring some gain anisotropy within the VCSEL cavity, and fix the VCSEL output polarization state regarding the LC orientation [6]. The second advance is to replace the LC material itself by a more robust in temperature one. In particular, we exploited for this work the properties of a LC presenting a higher clearing temperature (QYPDL36, with a $\mathrm{Tc}=93^{\circ} \mathrm{C}$ ) in comparison with the $\mathrm{LC}$ used in the first demonstration $\left(\mathrm{E} 7\right.$ with a $\left.\mathrm{Tc}=58^{\circ} \mathrm{C}\right)$.

\section{Results}

These VCSEL are characterized under optical pumping, at room temperature, and in CW operation. As shown on Fig.1, lasing operation is demonstrated, with typical threshold of 8-10 mW, with applied voltage on the LC micro-cells varying from $20 \mathrm{~V}$ down to $4.5 \mathrm{~V}$. These thresholds are very close to those obtained with single frequency VCSELs realized without any LC micro-cells. This is an experimental evidence than optical losses introduced by the LC inserted in the VCSEL cavity are significantly low. Considering our first 
demonstration, the main difference here lies on the fact that once the VCSEL has reached its threshold, its output polarization state remains almost fixed according to the LC extraordinary orientation, and thus all over pump power range, until the VCSEL emission switches off after the rollover. No more polarization switches, or bistability are observed in this new configuration. As shown on Fig. 2, for a 4.5V applied on the LC, the VCSEL emission is centered at $1561 \mathrm{~nm}$. By increasing the LC applied voltage up to $20 \mathrm{~V}$, a continuous blue shift of the VCSEL wavelength is obtained down to $1528 \mathrm{~nm}$, corresponding to a $33 \mathrm{~nm}$ wavelength tuning range. The larger tuning range and the better mode behavior are ascribed to the two main improvements we proposed. The respective contribution of the modifications of the active zone and the LC part will be further discussed to get better understanding on this significant improvement, and to draw up what could be future design rules for demonstrating the full potentialities of the LC-VCSEL in terms of tuning range and wavelength stability.

\section{Conclusion}

A tunable $1550 \mathrm{~nm}$ InP VCSEL has been designed and realized, integrating a liquid crystal micro-cell. CW operation is demonstrated at room temperature, and improved performances are demonstrated in terms of wavelength tuning $(33.3 \mathrm{~nm})$, and VCSEL emission stability all over the excitation range. These improvements are mainly related with a better temperature management of the LC intracavity layer, and the VCSEL polarization state control exploiting a section with QDHs. Further improvements in terms of output power and wavelength tuning are expected.

The authors acknowledge RENATECH+ (the French national network of facilities for micronanotechnology) with LAAS-CNRS, C2N-CNRS/UPSUD and NanoRennes for technological support. The Direction Générale de l'Armement (DGA) and the French National Research Agency (ANR) are acknowledged for financial support (ANR-14-ASTR-0007 HYPOCAMP and ANR-15-CE19-0012 DOCT VCSEL)).

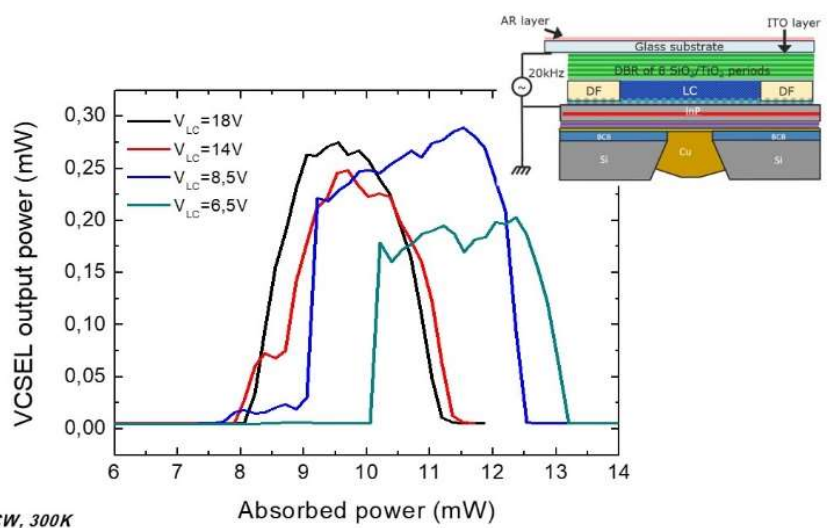

Fig. 1. VCSEL output power as function of pump absorbed power for different voltages applied on the LC $\mu$ cell $(20 \mathrm{kHz})$. Insert is a schematic of the LC $\mu$ cell VCSEL

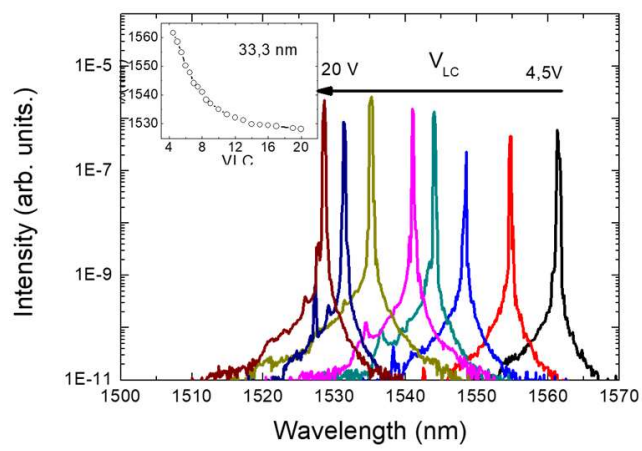

Fig. 2. VCSEL spectra measured at pump power of $10 \mathrm{~mW}$, for different LC applied voltages $(20 \mathrm{kHz})$. Insert shows the VCSEL lasing wavelength as function of the LC applied voltage.

\section{References}

[1] P. Qiao, K. T. Cook, K. Li, et C. J. Chang-Hasnain, « Wavelength-Swept VCSELs », IEEE J. Sel. Top. Quantum Electron., vol. 23, no 6, p. 1-16, nov. 2017, doi: 10.1109/JSTQE.2017.2707181

[2] C. Belmonte et al., « Optimization of electrically tunable VCSEL with intracavity nematic liquid crystal », Opt. Express, vol. 23, no 12, p. 15706, juin 2015, doi: 10.1364/OE.23.015706.

[3] B. Sadani et al., « Liquid-Crystal Alignment by a Nanoimprinted Grating for Wafer-Scale Fabrication of Tunable Devices », IEEE Photonics Technol. Lett., vol. 30, no 15, p. 1388-1391, août 2018, doi: 10.1109/LPT.2018.2849641.

[4] C. Levallois et al., " Liquid crystal-based tunable photodetector operating in the telecom C-band », Opt. Express, vol. 26, no 20, p. 25952, oct. 2018, doi: 10.1364/OE.26.025952.

[5] B. Boisnard et al., "CW Operation of a Tunable 1550-nm VCSEL Integrating Liquid-Crystal Microcells », IEEE Photonics Technol. Lett., vol. 32, no 7, p. 391-394, avr. 2020, doi: 10.1109/LPT.2020.2975076

[6] J. M. Lamy et al, « Polarization control of $1.6 \mu \mathrm{m}$ vertical-cavity surface-emitting lasers using InAs quantum dashes on InP(001) », Applied Phys. Lett. , vol 95, p. 011117, 2009, doi : 10.1063/1.3176437 